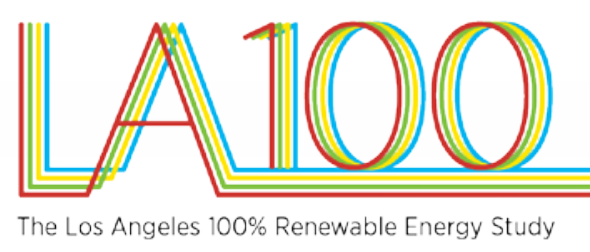

\title{
The Los Angeles
}

\section{0\% Renewable Energy Study (LA100)}

Rob Leland, PhD

Associate Laboratory Director for Scientific Computing and Energy Analysis National Renewable Energy Laboratory June 2020 


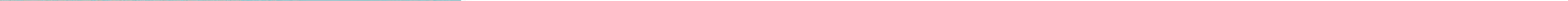


What Is Unique About LA100?

\section{$\Pi \rrbracket$}

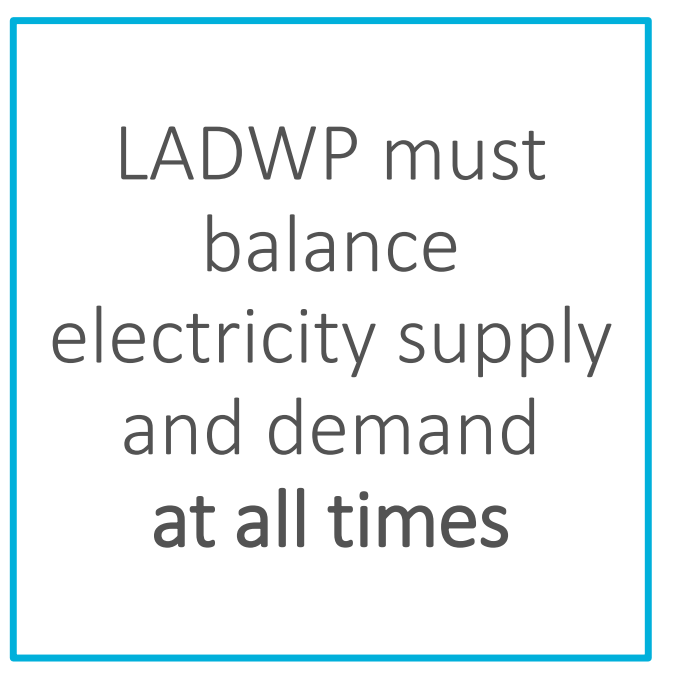

First-of-its-kind, ultrahigh resolution modeling

\section{Objective,} transparent, stakeholder-based analysis of pathways to 100\% RE 


\section{Scenarios Based on LA Advisory Group Priorities}

\begin{tabular}{|c|c|c|c|c|c|c|c|c|c|c|}
\hline & & \multicolumn{9}{|c|}{ LA100 Scenarios } \\
\hline & & \multicolumn{4}{|c|}{ Moderate Load Electrification } & \multicolumn{4}{|c|}{ High Load Electrification (Load Modernization) } & \multirow{2}{*}{$\begin{array}{c}\text { High Load Stress } \\
\text { S8100 }\end{array}$} \\
\hline & & SB100 & $\begin{array}{l}\text { LA-Leads, } \\
\text { Emisslons Free (No } \\
\text { Biofuels) }\end{array}$ & $\begin{array}{l}\text { Transmission } \\
\text { Renalissance }\end{array}$ & $\begin{array}{l}\text { High Distributed } \\
\text { Energy Future }\end{array}$ & SB100 & $\begin{array}{l}\text { LA-Leads, } \\
\text { Emissions Free (No } \\
\text { Biofuels) }\end{array}$ & $\begin{array}{l}\text { Transmission } \\
\text { Renaissance }\end{array}$ & $\begin{array}{l}\text { High Distributed } \\
\text { Energy Future }\end{array}$ & \\
\hline & RE Target in $\mathbf{2 0 3 0}$ with RECs & $60 \%$ & $100 \%$ & $100 \mathrm{~K}$ & $100 \%$ & $60 \%$ & $100 \%$ & $100 \%$ & $100 \times$ & $60 \%$ \\
\hline & Compllance Year for $100 \%$ RE & 2045 & 2035 & 2045 & 2045 & 2045 & 2035 & 2045 & 2045 & 2045 \\
\hline \multirow{8}{*}{$\begin{array}{l}\text { Technologies that } \\
\text { do not vary in } \\
\text { eligibility across } \\
\text { scenarios }\end{array}$} & Solid Biomass & $\mathrm{N}$ & $\mathrm{N}$ & $\mathrm{N}$ & N & $\mathrm{N}$ & $\mathrm{N}$ & $\mathrm{N}$ & $\mathrm{N}$ & $\mathrm{N}$ \\
\hline & Fuel Cells & $\mathrm{r}$ & Y & $\mathrm{Y}$ & $\mathrm{Y}$ & $\mathrm{Y}$ & $\mathrm{Y}$ & $Y$ & $\mathrm{Y}$ & $\gamma$ \\
\hline & Hydro - Existing & $\gamma$ & $Y$ & $\mathrm{Y}$ & 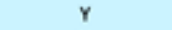 & $\mathrm{Y}$ & $\mathrm{Y}$ & $Y$ & $\mathrm{Y}$ & $\mathrm{Y}$ \\
\hline & Hydro - New & $\mathrm{N}$ & $\mathrm{N}$ & $\mathrm{N}$ & $\mathrm{N}$ & $\mathrm{N}$ & $\mathrm{N}$ & $\mathrm{N}$ & $\mathrm{N}$ & $\mathrm{N}$ \\
\hline & Hydro - Upgrades & $\gamma$ & r & $\mathrm{r}$ & $\gamma$ & $\mathrm{r}$ & $\gamma$ & $\gamma$ & $\mathrm{Y}$ & $\gamma$ \\
\hline & Nuclear - New & $\mathrm{N}$ & $\mathrm{N}$ & $\mathrm{N}$ & $\mathrm{N}$ & $\mathrm{N}$ & $\mathrm{N}$ & $\mathrm{N}$ & N & $\mathrm{N}$ \\
\hline & Wind, Solar, Geothermal & r & r & $\mathrm{r}$ & Y & $\mathrm{Y}$ & Y & r & $\mathrm{Y}$ & $\mathrm{r}$ \\
\hline & Storage & Y & Y & $\mathrm{Y}$ & Y & $\mathrm{Y}$ & $\mathrm{Y}$ & Y & $\mathrm{Y}$ & $\mathrm{Y}$ \\
\hline \multirow{4}{*}{$\begin{array}{c}\text { Technologies that } \\
\text { do vary }\end{array}$} & Biofuel Combustion & $Y$ & No & $\mathrm{Y}$ & $Y$ & $\mathrm{Y}$ & No & $Y$ & $\mathrm{Y}$ & $\mathrm{Y}$ \\
\hline & RE-derived Fuel Combustion (e.g., hydrogen) & r & No & $\mathrm{Y}$ & $\gamma$ & $\mathrm{Y}$ & No & $\gamma$ & $\mathbf{Y}$ & $\gamma$ \\
\hline & Natural Gas & $\gamma$ & No & No & No & $\mathrm{Y}$ & No & No & No & $\gamma$ \\
\hline & Nuclear - Existing & $\gamma$ & $r$ & No & No & $\mathrm{r}$ & $\mathrm{Y}$ & No & No & $\gamma$ \\
\hline Repowering OTC & Haynes, Scattergood, Harbor & $\mathrm{N}$ & $\mathrm{N}$ & $\mathrm{N}$ & $\mathrm{N}$ & $\mathrm{N}$ & $\mathrm{N}$ & $\mathrm{N}$ & $\mathrm{N}$ & $\mathrm{N}$ \\
\hline RECS & $\begin{array}{l}\text { Financial Mechanisms } \\
\text { (RECS/Allowances) }\end{array}$ & Yes & $\mathrm{N}$ & $\mathrm{N}$ & $\mathrm{N}$ & Yes & $\mathrm{N}$ & $\mathrm{N}$ & $\mathrm{N}$ & Yes \\
\hline DG & Distributed Adoption & Moderate & Hizh & Moderate & Hich & Moderate & High & Moderate & High & Moderate \\
\hline Load & $\begin{array}{l}\text { Energy Efficiency } \\
\text { Demand Response } \\
\text { Electrification }\end{array}$ & $\begin{array}{l}\text { Moderate } \\
\text { Moderate } \\
\text { Moderate }\end{array}$ & $\begin{array}{l}\text { Moderate } \\
\text { Moderate } \\
\text { Moderate }\end{array}$ & $\begin{array}{l}\text { Moderate } \\
\text { Moderate } \\
\text { Moderate }\end{array}$ & $\begin{array}{l}\text { Moderate } \\
\text { Moderate } \\
\text { Moderate }\end{array}$ & $\begin{array}{l}\text { High } \\
\text { High } \\
\text { High }\end{array}$ & $\begin{array}{l}\text { High } \\
\text { High } \\
\text { High }\end{array}$ & $\begin{array}{l}\text { High } \\
\text { High } \\
\text { High }\end{array}$ & $\begin{array}{l}\text { High } \\
\text { High } \\
\text { High }\end{array}$ & $\begin{array}{c}\text { Reference } \\
\text { Reference } \\
\text { High }\end{array}$ \\
\hline Transmission & $\begin{array}{l}\text { New or Upgraded Transmission } \\
\text { Allowed? }\end{array}$ & $\begin{array}{l}\text { Only Along Existing } \\
\text { or Planned } \\
\text { Corridors }\end{array}$ & $\begin{array}{l}\text { Only Along Existing } \\
\text { or Planned } \\
\text { Corridors }\end{array}$ & $\begin{array}{l}\text { New } \\
\text { Corridors } \\
\text { Allowed }\end{array}$ & $\begin{array}{l}\text { No New } \\
\text { Transmission }\end{array}$ & $\begin{array}{l}\text { Only Along Existing } \\
\text { or Planned } \\
\text { Corridors }\end{array}$ & $\begin{array}{l}\text { Only Nlong Existing } \\
\text { or Planned } \\
\text { Corridors }\end{array}$ & $\begin{array}{l}\text { New } \\
\text { Corridors } \\
\text { Allowed }\end{array}$ & $\begin{array}{l}\text { No New } \\
\text { Transmission }\end{array}$ & $\begin{array}{l}\text { Only Nong Existing } \\
\text { or Planned } \\
\text { Corridors }\end{array}$ \\
\hline WECC & WECC VRE Penetration & Moderate & Moderate & Moderate & Moderate & Moderate & Moderate & Moderate & Moderate & Moderate \\
\hline
\end{tabular}

Note, the study also includes a reference case (2017 IRP with minor updates). This case extends through 2036. 
dGen

Modeled every property in

LA (625,291 agents),

totaling $>65 \mathrm{M}$ simulations

Buildings

Modeled $>7 \mathrm{M}$ buildings using 3.6M processor hours, which would take $>60$ years to finish on a laptop

\section{dsgrid}

Allocated 5 modeling teams' loads to 625,291 geographic locations, generating $>3.5 \mathrm{M}$ combinations and producing $\mathbf{5 0}$ TB of data; if stored in CDs, this would be taller than a 16-story building

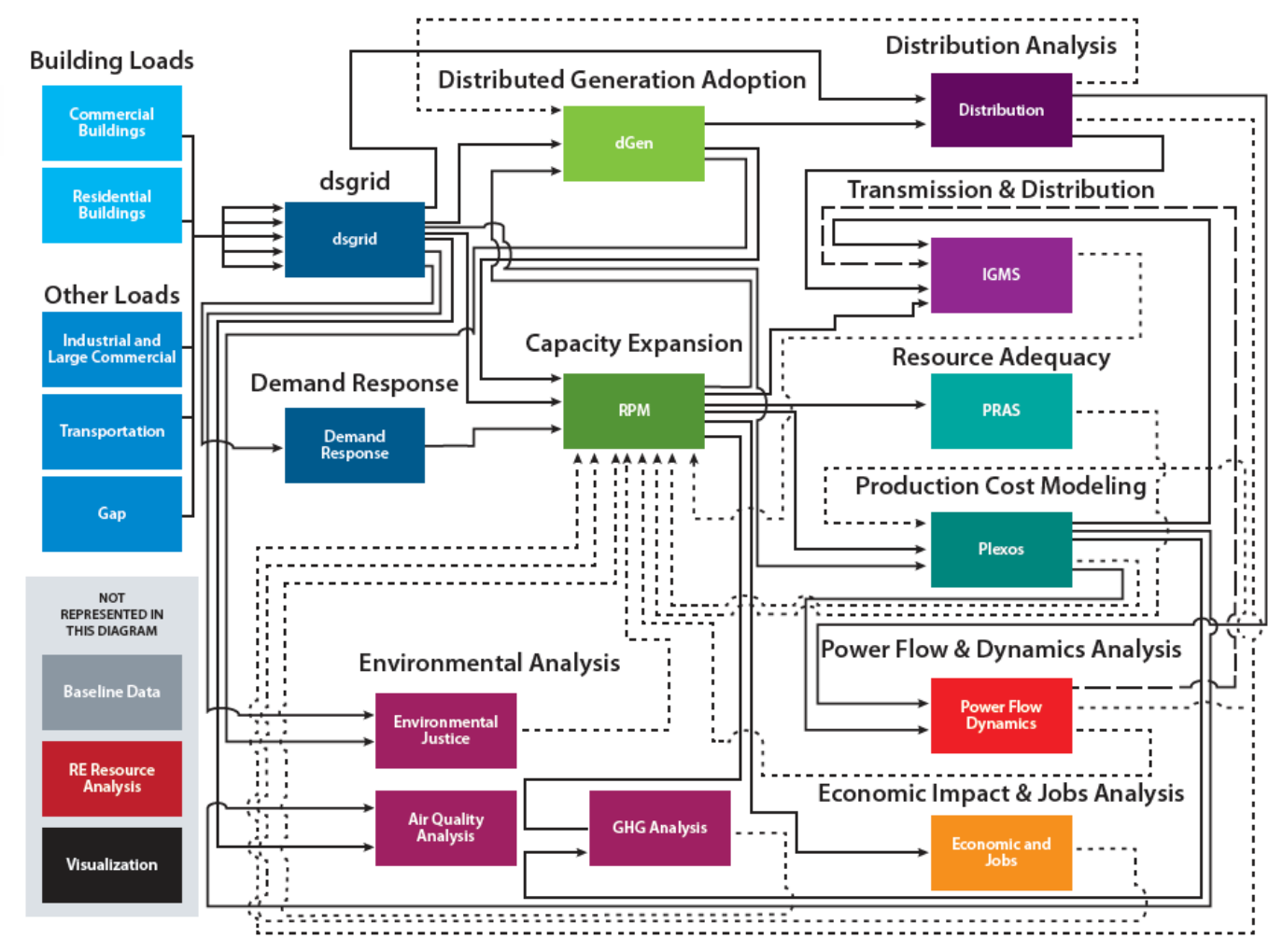

\section{Distribution}

Modeled every electric wire in LA (over $\mathbf{1 , 6 0 0}$ circuits) for thousands of scenarios each-totaling >25M detailed engineering simulations

\section{RPM}

Simulated $>8,000$ years of dispatch , which would require $\mathbf{2}$ decades worth of computing on a laptop

\section{Plexos}

Ran >7.6 node-years on Eagle, which is like conducting a simulation 24/7 for 7.6 years straight on a laptop 


\section{Example of results: Transmission Planning}

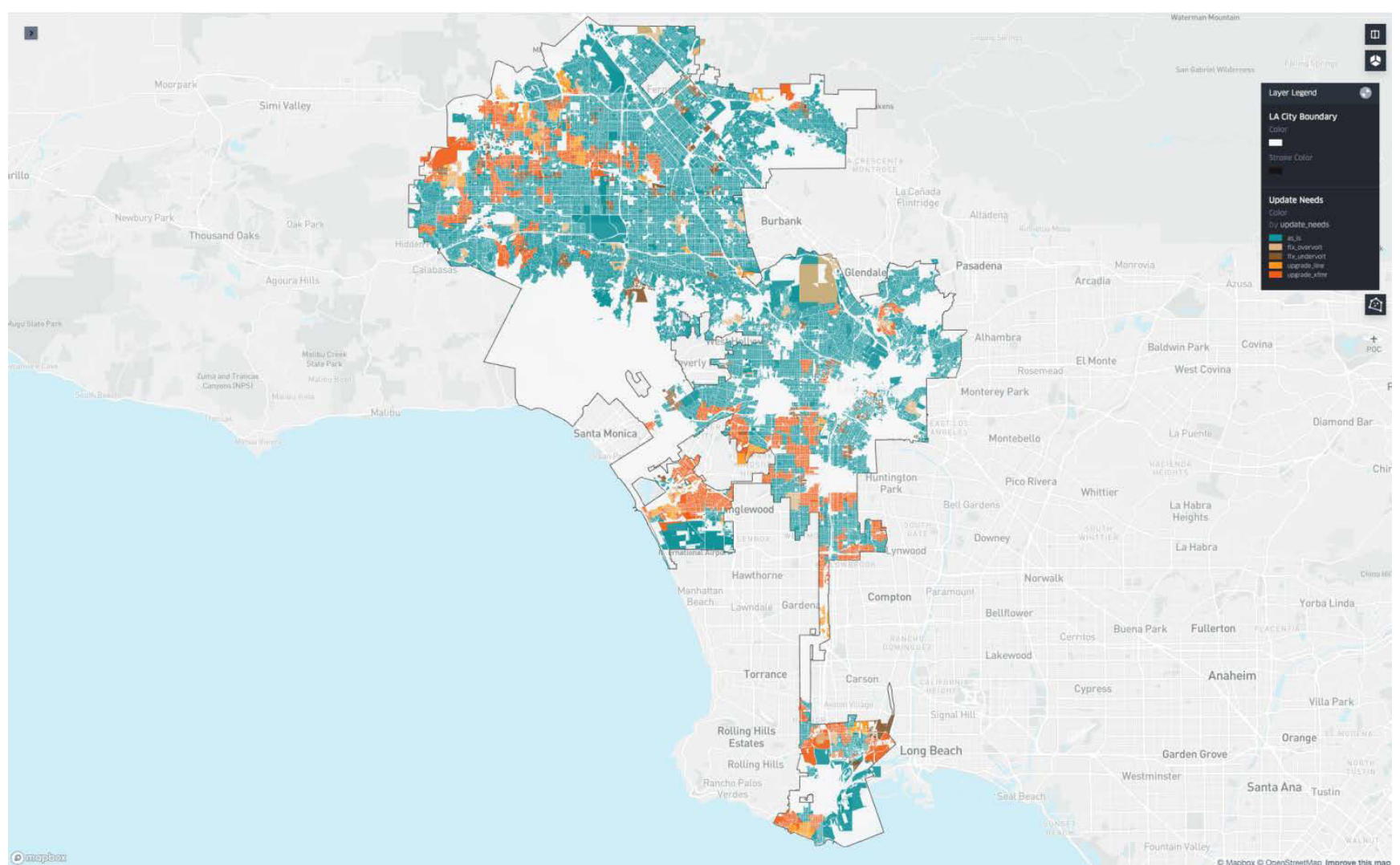


1. Multiple cost-effective pathways to get to $\sim 90 \% R E$

2. Last $10 \%$ will likely employ new technologies

3. Many uncertainties about the last $10 \%$, but the broader the eligible solutions, the lower the costs

4. Cross-economy impacts require careful analysis 
- LA100 offers unprecedented scale, scope, and detail for energy planning

But next analyses will be even more complex as we further integrate modeling

\section{Closing \\ Thoughts}

- High-performance computing - at the scale of a national laboratory-is essential to provide robust insights

- The study findings help identify characteristics of new technologies to meet market demand as more cities and states adopt $100 \%$ clean energy targets 


\section{Thank you!}

\section{NREL/PR-6A70-77043}

This work was authored by the National Renewable Energy Laboratory, operated by Alliance for Sustainable Energy, LLC, for the U.S. Department of Energy (DOE) under Contract No. DE-AC36-08GO28308. Funding provided by the U.S. Department of Energy for Energy Analysis \& Decision Support. The views expressed in the article do not necessarily represent the views of the DOE or the U.S. Government. The U.S. Government retains and the publisher, by accepting the article for publication, acknowledges that the U.S. Government retains a nonexclusive, paid-up, irrevocable, worldwide license to publish or

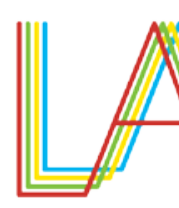
reproduce the published form of this work, or allow others to do so, for U.S. Government purposes. 